Christian Sickel

Verkaufsfaktor Kundennutzen 


\section{Christian Sickel}

\section{Verkaufsfaktor Kundennutzen}

Konkreten Bedarf ermitteln, aus Kundensicht argumentieren, maßgeschneiderte Lösungen präsentieren

4., erweiterte Auflage

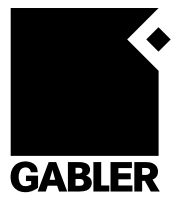


Bibliografische Information Der Deutschen Nationalbibliothek

Die Deutsche Nationalbibliothek verzeichnet diese Publikation in der

Deutschen Nationalbibliografie; detaillierte bibliografische Daten sind im Internet über <http://dnb.d-nb.de> abrufbar.

Das Buch erschien in der 1. Auflage unter dem Titel "Ohne Nutzen kein Verkauf".

1. Auflage 1999

2., erweiterte Auflage 2003

3., erweiterte Auflage 2006

4., erweiterte Auflage 2008

Alle Rechte vorbehalten

(C) Betriebswirtschaftlicher Verlag Dr. Th. Gabler I GWV Fachverlage GmbH, Wiesbaden 2008

Lektorat: Manuela Eckstein

Der Gabler Verlag ist ein Unternehmen von Springer Science+Business Media. www.gabler.de

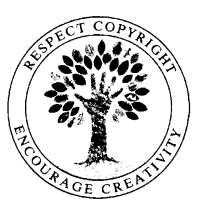

Das Werk einschließlich aller seiner Teile ist urheberrechtlich geschützt. Jede Verwertung außerhalb der engen Grenzen des Urheberrechtsgesetzes ist ohne Zustimmung des Verlags unzulässig und strafbar. Das gilt insbesondere für Vervielfältigungen, Übersetzungen, Mikroverfilmungen und die Einspeicherung und Verarbeitung in elektronischen Systemen.

Die Wiedergabe von Gebrauchsnamen, Handelsnamen, Warenbezeichnungen usw. in diesem Werk berechtigt auch ohne besondere Kennzeichnung nicht zu der Annahme, dass solche Namen im Sinne der Warenzeichen- und Markenschutz-Gesetzgebung als frei zu betrachten wären und daher von jedermann benutzt werden dürften.

Umschlaggestaltung: Nina Faber de.sign, Wiesbaden

Satz: ITS Text und Satz Anne Fuchs, Pfofeld-Langlau

Druck und buchbinderische Verarbeitung: Wilhelm \& Adam, Heusenstamm

Gedruckt auf säurefreiem und chlorfrei gebleichtem Papier

Printed in Germany

ISBN 978-3-8349-0951-0 


\section{Vorwort}

Es genügt nicht, dass Unternehmen hervorragende Produkte herstellen, ausgezeichnete Dienstleistungen anbieten oder mit perfektem Service aufwarten. Allein mit diesen, noch so positiven Eigenschaften können Sie zwei entscheidende Fragen Ihres Gegenübers nicht beantworten: „Brauche ich das?“ und „Was nützt mir das?" Bedarf und Nutzen geben dem Verkauf erst einen Sinn. Beachten Sie bitte, dass hiermit nicht gemeint ist, was der Verkäufer als Bedarf oder Nutzen erkennt, sondern das, was der Kunde als solchen bejaht.

Oft wird - auch im Verkauf von Lösungen oder so genannten erklärungsbedürftigen Wirtschaftsgütern - angenommen, dass die Brücke zwischen Produkt und Bedarf der Preis sei. Irrtum! Der herausgearbeitete Nutzen, den ein Kunde erkennt, bestimmt den Wert eines Produkts oder einer Lösung. Die Idee, ein Produkt herzustellen oder eine Dienstleistung anzubieten, entspringt aus der Tatsache, dass es für den Nutzer einen Gewinn darstellt. Ein Erzeugnis, das einzig und allein existiert, weil es billig ist, gibt es auf dieser Welt nicht.

Immer noch existieren im Vertrieb zwei Lager: die Lösungs- und die Produktverkäufer. Die Lösungsverkäufer meinen, sie hätten es besonders schwer, weil sie dem Kunden nichts zum Anfassen zeigen können wie die Produktverkäufer. Die Produktverkäufer glauben, sie hätten es besonders schwer, weil ihre Produkte vergleichbarer sind als die der Lösungsverkäufer. In Wirklichkeit erschweren sich beide Lager ihren Verkauf. Die einen reden viel zu viel von ihren intelligenten Lösungen, die anderen zeigen viel zu viel von ihren tollen Produkten. Und das soll der Kunde dann super finden.

Nun gewinnt im Verkauf aber nicht derjenige, der objektiv betrachtet die bessere Lösung oder das bessere Produkt hat, son- 
dern derjenige, dem es gelingt, sein Angebot in den Augen des Kunden so zu positionieren, dass dieser es als das bessere empfindet.

Dabei ist es nebensächlich, was Sie verkaufen. Entscheidend ist immer, wie Sie es verkaufen. Denn:

Maßgeblich entscheidend für den Verkauf von

Produkten und Lösungen ist nicht, wie wunderbar und innovativ Sie sind. Ausschlaggebend ist, ob Sie eine Antwort auf das geben, was Ihre Kunden mehr oder weniger bewusst beschäftigt. Etwas, worauf die Kunden warten, ohne es manchmal selbst zu wissen.

Erst wenn Sie dem Kunden helfen, aus dem, was ihn beschäftigt, eine konkrete Bedarfssituation zu formulieren, haben Sie die erfolgreiche Basis für Ihren späteren Verkauf geschaffen.

Ein Bedarf ist immer eine Mangelsituation, eine Unzufriedenheit oder ein Problem. Wenn es Ihnen also gemeinsam mit Ihrem Kunden gelingt, seinen Bedarf zu erkennen und zu konkretisieren, haben Sie drei Dinge erreicht:

1. Der Kunde empfindet Sie als kompetent (wer ein Problem erkennt, dem traut man auch die Lösung zu).

2. Sie haben einen immensen Wettbewerbsvorsprung.

3. Der Preis Ihres Angebotes tritt in den Hintergrund.

Sie werden in diesem Buch damit vertraut gemacht, wie es gelingt, den Bedarf, den Sie bei jedem Kunden vermuten, zu einem für den Kunden konkreten Bedarf auszubauen. Mit den angeführten Beispielen wird es Ihnen später leicht fallen, den Mehrwert Ihres Angebots für den Kunden mit einer individuellen Nutzenargumentation sichtbar zu machen. 
Gestandene Verkäufer werden vielleicht milde lächelnd denken: „Aha, ein Buch für Anfänger!“ Trotzdem werden auch sie hier Übungen finden, deren Lösungen am Ende des Buches sie vermutlich überraschen werden.

Um Sie noch besser dabei zu unterstützen, sich zum wahren Nutzenverkäufer zu qualifizieren, habe ich dieser Auflage noch einige Umsetzungshilfen beigefügt. Sie finden Formulierungshilfen für Fragen und Übungen, die Sie allein oder im Team durchführen können. Denn Produkte und Lösungen gleichen sich. Der Kundennutzen hingegen ist immer individuell. Je mehr Sie den Bedarf des Kunden konkretisieren können, desto größer ist Ihre Chance, erfolgreich zu sein. Besonders in komplexen Verkaufssituationen, in denen Sie mit verschiedenen Gesprächspartnern auf unterschiedlichen Hierarchieebenen sprechen, wird deutlich, dass Ihre Verhandlungspartner neben dem geschäftlichen Nutzen auch immer einen persönlichen Gewinn aus Ihrem Angebot ziehen möchten.

Heute haben Sie es im Allgemeinen mit komplexen Verkaufssituationen zu tun. Sie müssen Ihr Augenmerk auf mehrere Personen im Kundenunternehmen richten, die entscheidenden Gesprächspartner identifizieren und für sie eine individuelle Nutzenargumentation erarbeiten. Überdies werden die Budgets bei Kunden immer kleiner, sodass neue Wege, aktiv auf den Kunden zuzugehen, immer erfolgsentscheidender werden. Und natürlich werden Sie erst vom Kunden als Lösungsverkäufer oder Problemlöser wahrgenommen, wenn Sie ihm mit der richtigen Verkaufsstrategie begegnen. Ich habe mich bemüht, diesen veränderten Einkaufsgewohnheiten von Unternehmen Rechnung zu tragen.

Jetzt wünsche ich Ihnen mit diesem Buch viel Spaß und bei der Umsetzung in Ihrem Verkaufsalltag viel Erfolg!

Köln, im März 2008

Ihr Christian SiCKEL 


\section{Inhalt}

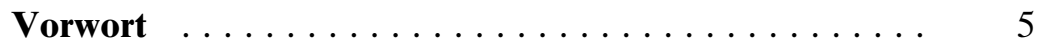

1. Einführung $\ldots \ldots \ldots \ldots \ldots \ldots \ldots \ldots \ldots \ldots$

Vermuteter und konkreter Bedarf .......... 11

Merkmal, Vorteil, Nutzen ............. 16

Was kennzeichnet die Nutzenargumentation im

Dienstleistungs- oder Lösungsverkauf? . . . . . . . 29

2. Die Methode ... . . . . . . . . . . . . . 35

Phase 1: Orientierungsfragen .......... 36

Phase 2: Problemfragen .............. 37

Phase 3: Auswirkungsfragen ........... 40

Phase 4: Lösungsfragen .............. 42

3. Praktische Anwendung . . . . . . . . . . . . . 49

Warum sollten Sie Fragen stellen, und wie wird Ihr

Verkauf hierdurch unterstützt? .......... 49

Wie unterscheiden sich die verschiedenen

Fragetypen? ................. 52

Wie Sie günstige und riskante Zeitpunkte für die verschiedenen Fragen erkennen . . . . . . . 56

Welche unterschiedlichen Möglichkeiten haben Sie, Ihre Fragen $\mathrm{zu}$ formulieren? . . . . . . . . . 66

Wen Sie was fragen sollten $\ldots \ldots \ldots \ldots \ldots$. 81

Die richtigen Fragen für den richtigen Kundentyp . 86

Ideen zur Umsetzung $\ldots \ldots \ldots \ldots \ldots \ldots$. . . . 91 
4. Die richtigen Gesprächspartner für Ihre Lösung

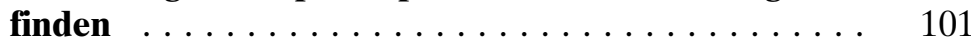

Die Nutzenargumentation in der Akquisitionsphase 106 Aufgabenstellung bei der Neukundenakquisition . . . 109

5. Welche Verkaufsstrategie den meisten Erfolg beschert .................... 117

6. Die Kundenorganisation besser durchdringen . . . 129

7. Die Phasen eines Kundenbesuchs $\ldots \ldots \ldots \ldots 133$

8. Die Zusage des Kunden erlangen $\ldots \ldots \ldots \ldots \ldots$

9. Der Nutzen und der Preis $\ldots \ldots \ldots \ldots 1$

10. Einwandbehandlung $\ldots \ldots \ldots \ldots \ldots 1$

Wie Sie Einwände vermeiden . . . . . . . . . 162

Kategorien von Einwänden . . . . . . . . . . 168

Vorgehensweise ................... 169

Die taktische Behandlung von Einwänden ...... 173

11. Der Umgang mit Vorwänden f . . . . . . . . . . . 187

Lösungen $\ldots \ldots \ldots \ldots \ldots \ldots \ldots \ldots \ldots \ldots \ldots \ldots$

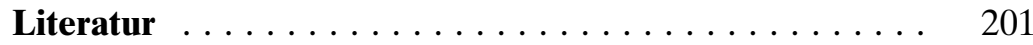

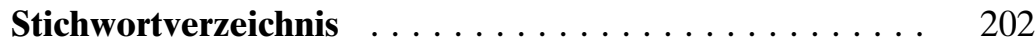

Der Autor $\ldots \ldots \ldots \ldots$. . . . . . . . . . . . . . . . . . . 204 